\section{Propriospinaler Myoklonus beim Einschlafen}

Helga Peter

Marburg, Deutschland

\section{Synonyme}

PSM

\section{Englischer Begriff}

propriospinal myoclonus at sleep onset (PSM)

\section{Definition}

Die Störung tritt in der Regel beim ruhenden, entspannten Patienten am Schlaf-Wach-Übergang auf, seltener bei Wach- phasen während des Schlafs oder beim Aufwachen. Sie verschwindet beim Erreichen eines stabilen Schlafstadiums oder durch mentale Aktivität. Die Betroffenen klagen über plötzliche Zuckungen von Bauch, Rumpf und Hals, die in vielen Fällen eine schwere Insomnie (siehe > „Insomnien“) verursachen, da sie das Einschlafen verhindern. Die Zuckungen nehmen ihren Ausgang von Muskeln, die von thorakalen und zervikalen spinalen Segmenten innerviert sind und breiten sich rostral und kaudal aus. EEG-Veränderungen im Zusammenhang mit den Zuckungen finden sich nicht. Die Pathophysiologie ist noch ungeklärt, prädisponierende oder auslösende Faktoren sind nicht bekannt. Die seltene Störung betrifft ausschließlich Erwachsene und verläuft chronisch. Die Patienten entwickeln häufig Angst vor dem Einschlafen, eventuell generalisierte Ängste oder Depressionen. Ein Zusammenhang mit dem Propriospinalen Myoklonus am Tage wird diskutiert.

Die > „ICSD-3“ (2014) zählt die Störung zu den Isolierten Symptomen und Normvarianten unter den Schlafbezogenen Bewegungsstörungen (siehe $>$,Schlafbezogene Bewegungsstörungen"). 